УДК 515.123

\title{
Об атомарности внешних мер
}

\author{
А.Н. Саженков ${ }^{1}$ Е.А. Плотникова ${ }^{2}$ \\ ${ }^{1}$ Алтайский государственный университет (Барнаул, Россия) \\ ${ }^{2}$ Новосибирский государственный технический университет (Новосибирск, \\ Россия)
}

\section{On Atomicity of External Measures}

\author{
A.N. Sazhenkov', E.A. Plotnikova ${ }^{2}$ \\ ${ }^{1}$ Altai State University (Barnaul, Russia) \\ ${ }^{2}$ Novosibirsk State Technical University (Novosibirsk, Russia)
}

Исследуется понятие неатомарности для абстрактных внешних мер, определенных на булевом кольце и принимающих значения в произвольном множестве. Для скалярных функций множеств неатомарность множества означает его представимость в виде объединения конечного числа попарно непересекающихся множеств, на любых принадлежащих кольцу подмножествах которых модуль значения функции будет меньше любого заранее заданного положительного числа.

Топологии, связанные с внешней мерой, рассматриваемые нами, являются согласованными со структурой булевого кольца и имеющими определенным образом составленную базу фильтра окрестностей нуля. В такой топологии операции конъюнкции, дизъюнкции, сложения и умножения являются равномерно непрерывными. В работах Л.Я. Савельева представлен обзор современного состояния результатов по обширному кругу вопросов, связанных с внешними мерами и топологиями.

Рассматриваются понятие абстрактной внешней меры, которое охватывает широкий класс мер, и понятие внешней топологии, даны два определения ограниченности в топологической абелевой группе и исследована взаимосвязь этих определений. В основных результатах работы понятие неатомарности меры характеризуется как свойство ограниченности области определения внешней меры в подходящей топологической группе.

Ключевые слова: внешняя мера, непрерывность, ограниченность, топология, атомарность меры.

\section{DOI 10.14258/izvasu(2019)4-17}

Введение. Исследуется понятие неатомарности для абстрактных внешних мер, определенных на булевом кольце и принимающих значения в произвольном множестве. Для скалярных функций множеств, т.е. для функций, определенных на кольце множеств
We consider the notion of non-atomicity for abstract exterior measures being defined on the Boolean ring and taking values in an arbitrary set. For scalar functions of sets, non-atomicity of a set means that it can be represented as a finite union of pairwise non-intersecting sets with the following property: for any belonging to the ring subset of any of these non-intersecting sets, absolute value of the scalar function applied to the subset is less than any afore given positive value.

In this work, associated with the exterior measure topologies are consistent with the structure of the Boolean ring and have the specially constructed filter base of neighborhoods of zero. In this topology of operations, conjunction, disjunction, addition, and multiplication operators are uniformly continuous. Survey of nowadays state of study of topics related to exterior measures and topologies can be found in the works of L. Y. Saveliev.

We focus on the notion of abstract exterior measure, which covers a wide class of measures. Also, we consider the notion of exterior topology. We set two definitions of boundedness in topological Abelian group and investigate interconnection of these definitions. In the main results of the work, the notion of nonatomicity of measure is characterized as the property of boundedness of the domain of definition of exterior measure in an appropriate topological group.

Key words: exterior measure, continuity, boundedness, topology, atomicity of measure. и принимающих значения в числовой прямой, неатомарность множества $A$ означает, что его можно представить в виде объединения конечного числа попарно непересекающихся множеств $A_{1}, A_{2}, \ldots, A_{n}$ таких, что для любого номера $k$ и любого подмножества 
$B \subset A_{k}$ из кольца модуль значения функции на этом множестве будет меньше заранее заданного положительного числа $\varepsilon$.

В первой части работы вводятся понятие абстрактной внешней меры, которое охватывает широкий класс мер, и понятие внешней топологии. Во второй даны два определения ограниченности в топологической абелевой группе. Рассмотрена взаимосвязь этих определений. В заключительной части приведены основные результаты работы. Здесь понятие неатомарности характеризуется как свойство ограниченности области определения внешней меры в подходящей топологической группе.

Внешние меры и топологии. Основные определения. Пусть $(P, \vee, \wedge, C)-$ булева алгебра, $(R,+, \cdot)$ - булево подкольцо $P, 0$ - минимальный элемент $P$ [1]. $M$ - произвольное множество, на котором выделена точка $\theta, U-$ фильтр по убыванию его подмножеств, содержащих точку $\theta . \varphi-$ отображение $R$ в $M$ такое, что $\varphi(0)=\theta$, определим

$$
\tilde{\varphi}(x)=\{\varphi(y): y \leq x, y \in R\} \text { и } \tilde{u}=\{x \in R: \tilde{\varphi}(x) \subset u\} .
$$

Отображение $\varphi: R \rightarrow M$ будем называть внешней мерой, если для любого $u \in U$ существует $v \in U$ такое, что из $x, y \in R$ и $\tilde{\varphi}(x) \subset v, \tilde{\varphi}(y) \subset v$ следует $\tilde{\varphi}(x \vee y) \subset u$.

Нетрудно заметить, что в терминах операции + это определение эквивалентно следующему: для любого $u \in U$ существует $v \in U$ такое, что $\tilde{v}+\tilde{v} \subset \tilde{u}$. Данное определение охватывает широкий класс мер. Это, например, классические внешние меры, т.е. функции $\mu$ со свойствами: $\mu(\varnothing)=0, \mu(A) \geq 0, \mu(A) \leq \mu(B)$, если $A \in B, \mu(A \cup B) \leq \mu(A)+\mu(B)$, которые удовлетворяют определению внешней меры для фильтра

$U=\{[0, \varepsilon): \varepsilon>0\}, \theta=0$.

Нетрудно заметить также, что если $M-$ абелева группа, то любая конечно аддитивная функция, т.е. функция, обладающая свойством: из $x \wedge y=0$ следует $\varphi(x \vee y)=\varphi(x)+\varphi(y)$, является внешней мерой, какой бы ни был фильтр $U$.

Для каждой внешней меры $\varphi$ существует единственная, согласованная со структурой кольца $(R,+, \cdot)$ топология $I(\varphi)$, для которой $\{\tilde{u}: u \in U\}$ составляют базу фильтра окрестностей 0 . Операции $\vee, \wedge,+, \cdot$ равномерно непрерывны при топологии $I(\varphi)[2,3,4]$. В частности, $(R,+, I(\varphi))$ является топологической абелевой группой. Топология $I(\varphi)$ называется жордановой внешней топологией. В работах [5-6] представлен обзор результатов по внешним мерам и топологиям.

Последовательность $\left\{x_{n}\right\} \subset P$ называется $\partial и з$ юнктной, если $x_{k} \wedge x_{m}=0$ при $k \neq m$. Функция $\varphi: R \rightarrow M$ называется исчерпывающеци, если для любого $v \in U$ и любой дизъюнктной последовательности $\left\{x_{n}\right\} \subset P$ найдется номер $N$ такой, что для всех $n \geq N$ выполня- ются включения $\varphi\left(x_{n}\right) \subset v$. Относительно исчерпывающих функций множеств в последнее время в литературе представлены работы Т.А. Срибной [7-8], в которых изучается свойство равномерной исчерпываемости семейств внешних мер. В данной работе для исчерпывающих внешних мер рассматриваются понятия атомарности - неатомарности и проводятся исследования по выявлению их свойств. Атомарность мер с различных точек зрения исследуется, например, в работах следующих авторов: E. Pap, A. Gavrilut, M. Agop, P. Cavaliere, F. Ventriglia [9-10].

Внешнюю меру $\varphi: R \rightarrow M$ будем называть неатомарной, если для любых $x \in R, v \in U$ существует дизъюнктный набор $x_{1}, x_{2}, \ldots, x_{n}$ элементов из $R$ такой, что $x=x_{1} \vee \ldots \vee x_{n}$ и $\tilde{\varphi}\left(x_{k}\right) \subset v$ для всех $k$. Отметим следующее свойство окрестностей $\tilde{u}$ элемента 0 топологии $I(\varphi)$ : если $x \in \tilde{u}, y \leq x$, то $y \in \tilde{u}$.

Ограниченность в топологических группах. В работах, исследующих свойства мер со значениями в группах, наиболее часто употребляются следующие определения ограниченности.

Определение 1. Подмножество $Q$ топологической абелевой группы $G$ называется ограниченным, если для любой $V$ окрестности нуля в $G$ существует натуральное число $n$ такое, что $Q \subset V+\ldots+V(n$ слагаемых). В дальнейшем $n$-кратную сумму окрестностей записываем так: $\stackrel{n}{+} V$.

Когда $G$ - топологическое векторное пространство, это определение эквивалентно классическому. Однако существенным недостатком такого определения является тот факт, что конечные множества в менее квалифицированных случаях могут оказаться неограниченными. Например, в дискретной группе, состоящей из двух и более элементов, единственным ограниченным множеством будет ноль. Определение ограниченности, предложенное Н. Бурбаки [4], указанного недостатка не имеет.

Определение 2. Подмножество $Q$ топологической абелевой группы $G$ называется ограниченным, если для любой $v$ окрестности нуля в $G$ существует натуральное число $n$ и конечный набор элементов группы $g_{1}, \ldots, g_{k}$ такие, что $Q \subset \bigcup_{i=1}^{k}\left(g_{i}+{ }^{n}+v\right)$.

В дальнейшем, чтобы различать виды ограниченности, будем называть подмножество Q 1-ограниченным (2-ограниченным), если оно удовлетворяет соответствующему определению.

Отметим некоторые свойства введенных понятий ограниченности, непосредственно следующие из определений и примера: 1-ограниченность множества влечет его 2-ограниченность; конечные множества являются 2-ограниченными; в дискретной группе, состоящей из двух и более элементов, наборы ограниченных множеств различны. 
Заметим, что если $Q$ является 2-ограниченным, то для симметричной, т.е. $v=-v$ окрестности нуля элементы $g_{1}, \ldots, g_{k}$ и натуральное $n$ можно выбрать так, что $g_{1}=0, g_{i} \in Q, g_{i} \notin \stackrel{m}{+} v$ для любого $m$ и $i=2, \ldots, k$.

Пусть $Q$ является 2-ограниченным, $Q \subset \bigcup_{i=1}^{r}\left(a_{i}+\stackrel{s}{+v}\right)$. Можно считать, что $Q \cap\left(a_{i}+\stackrel{s}{+v}\right) \neq \varnothing$, иначе удалим множества, не участвующие в покрытии. Для любого $a$ имеются две возможности: или $a_{i} \notin \stackrel{m}{+}{ }^{v}$ для любого $m$, или существует натуральное число $m_{i}$ такое, что $a_{i} \in+v$. В первом случае выберем $b_{i} \in Q \cap\left(a_{i}+\stackrel{s}{+} v\right) \neq \varnothing$, тогда $a_{i} \in b_{i}+\stackrel{s}{+}(-v) a_{i}+\stackrel{s}{+} v \subset b_{i}+\stackrel{2 s}{+v}, \quad$ кроме того, $b_{i} \notin \stackrel{m}{+} v$ для любого $m$. Переобозначим появившиеся элементы $b_{i}$ как $g_{2}, \ldots, g_{k}$. Во втором случае имеем: $a_{i}+\stackrel{s}{+} v \subset^{m_{i}+s}+v$. Обозначим $g_{1}=0, n-$ максимальное из найденных чисел $2 s$ и $s+m_{i}$. Таким образом,

$Q \subset \bigcup_{i=1}^{k}\left(g_{i}+\stackrel{n}{+} v\right)$.

Связь неатомарности внешней меры и ограниченности ее области определения в жордановой внешней топологии

Пусть внешняя мера $\varphi$ определена на булевом кольце $R$. Как было замечено в $\$ 1,(R,+, I(\varphi))$ является топологической абелевой группой. Приведенная ниже теорема характеризует понятие неатомарности как топологическое свойство множества $R$ в топологической абелевой группе $(R,+, I(\varphi))$.

Теорема 1. Пусть $\varphi-$ исчерпывающая внешняя мера. Тогда мера неатомарна, если и только если множество $R$ 1-ограничено в топологической абелевой группе $(R,+, I(\varphi))$.

Доказательство. Необходимость. Во-первых, заметим, что для любого $u \in U$ существует $x \in R$ такой, что для любого $y \in R$, дизъюнктивного с $x$, справедливо включение $\varphi(y) \in u$. Допустим, что это не так. Тогда для $x_{0}=0$ существует элемент $x_{1} \in R$ такой, что $\varphi\left(x_{1}\right) \notin u$. Для $x_{1}$ существует $x_{2}$ со свойствами: $x_{1}$ $\wedge x_{2}=0, \varphi\left(x_{2}\right) \notin u$. Далее, для $x_{1} \vee x_{2}$ найдется $x_{3}$ такой, что $\left(x_{1} \vee x_{2}\right) \wedge x_{3}=0, \varphi\left(x_{3}\right) \notin u$ и так далее... Получим дизъюнктивную последовательность $\left\{x_{n}\right\}$, для которой $\varphi\left(x_{n}\right) \notin u$, противоречие с исчерпываемостью.

Во-вторых, воспользуемся теперь неатомарностью внешней меры $\varphi$ и для найденного на первом шаге эле- мента $x$, найдем набор дизъюнктивных элементов $x_{1}, \ldots, x_{n}$ таких, что $x=\bigvee_{k=1}^{n} x_{k}$ и $\tilde{\varphi}\left(x_{k}\right) \subset u$, т.е. $x_{k} \in \tilde{u}$.

Пусть теперь $z \in R$ произвольный элемент. Обозначим $z_{0}=z+x z, z_{k}=z x_{k}$ для $k=1,2, \ldots, n$. Тогда получим $z=z_{0}+z_{1}+\ldots z_{n}$ и $z_{k} \in \tilde{u}$ для $k=0,1,2, \ldots$, $n+1$

$n$ и $z \in+\tilde{u}$. Отметим, что $n$ определено через $x$ и является одним для любых $z$.

Достаточность. Пусть $u \in U,-$ произвольное, тогда, по условию теоремы, существует $n$ такое, что $R \subset^{n}+\tilde{u}$. Рассмотрим произвольное $x \in R$, ясно $x \in \stackrel{n}{+} \tilde{u}$ и $x=x_{1}+\ldots+x_{n}$, где $x_{k} \in \tilde{u}, k=1,2, \ldots, n$. Теперь достаточно построить $z_{1}, \ldots, z_{n}$ такие, что: 1) $z_{i} \in \tilde{u}, i=1,2, \ldots, n$; 2) $z_{i} \wedge z_{j}=0(i \neq j)$; 3) $x=z_{1} \vee z_{2}$ $\vee \ldots \vee z_{n}$.

Проведем доказательство индукцией по $n$. Пусть $n=2$, т.е. $x=x_{1}+x_{2}, x_{1} \in \tilde{u}, x_{2} \in \tilde{u}$. Положим $z_{1}=x_{1}+x_{1} x_{2}, z_{2}=x_{2}+x_{1} x_{2}$ и получим $z_{1} \wedge z_{2}=z_{1} z_{2}=0$ и так как $z_{1} \leq x_{1}, z_{2} \leq x_{2}$, получим $z_{1} \in \tilde{u}, z_{2} \in \tilde{u}$. Пусть утверждение справедливо для $n=k$. Докажем его для $n=k+1$. Пусть $x=x_{1}+\ldots+x_{k}+x_{k+1}$, положим $t=x_{1}+\ldots+x_{k}$. По индукционному предположению существует дизъюнктивный набор $y_{1}, \ldots y_{k}$ такой, что $t=y_{1} \vee \ldots \vee y_{k}$, $y_{i} \in \tilde{u}$. Определим набор $\left\{z_{i}\right\}$ равенствами: $z_{i}=y_{i}+x_{k+1} y_{i}, i=1,2, \ldots, k ; z_{k+1}=x_{k+1}+t x_{k+1}$ и заметим, что он удовлетворяет свойствам 1-3. Во-первых, так как $z_{i} \leq y_{i}, y_{i} \in \tilde{u}$, получим $z_{i} \in \tilde{u}$, аналогично $z_{k+1} \leq x_{k+1}$, следовательно, $z_{k+1} \in \tilde{u}$. Во-вторых, так как $z_{i} \leq y_{i}, i=1,2, \ldots, k$ и $y_{i}$ попарно дизъюнктивны, получаем дизъюнктивность набора $z_{1}, z_{2}, \ldots, z_{k}$.

Поскольку $z_{i} z_{k+1}=\left(y_{i}+x_{k+1} y_{i}\right)\left(x_{k+1}+t x_{k+1}\right)=$ $=y_{i} x_{k+1}+x_{k+1}^{2} y_{i}+y_{i} t x_{k+1}+t y_{i} x_{k+1}^{2}$

и $y_{i} x_{k+1}+x_{k+1}^{2} y_{i}=y_{i} x_{k+1}+x_{k+1} y_{i}=0$,

$y_{i} t x_{k+1}+t y_{i} x_{k+1}^{2}=t \cdot\left(y_{i} x_{k+1}+y_{i} x_{k+1}\right)=t \cdot 0=0$, полу-

чаем $z_{i} z_{k+1}=0$. В-третьих, воспользуемся свойством булевых операций: если $a \wedge b=0$, то $a \vee b=a+b$, и получим цепочку равных выражений: $z_{1} \vee \ldots \vee z_{k} \vee z_{k+1}$, $z_{1}+\ldots+z_{k}+z_{k+1}, z_{1}+\ldots+z_{k}+z_{k+1},\left(y_{1}+y_{1} z_{k+1}\right)+\ldots+$ $+\left(y_{k}+y_{k} z_{k+1}\right)+\left(x_{k+1}+x_{k+1} t\right)$. Поскольку $y_{i}$ попарно дизъюнктивны, $t=y_{1}+\ldots y_{k}$, цепочку можно продолжить: $t+t x_{k+1}+x_{k+1}+x_{k+1} t=t+x_{k+1}=x_{1}+\ldots x_{k}+x_{k+1}$. Теорема доказана.

Замечание 1. При доказательстве достаточности не использовалась исчерпываемость внешней меры $\varphi$. Требование исчерпываемости в обратном утверждении не может быть опущено.

Пример. Пусть $R$ - кольцо множеств (оно булево относительно операций симметричной разности $\Delta$ 
и пересечения $\cap$ ), состоящее из ограниченных измеримых по Лебегу подмножеств $R ; \varphi-$ мера Лебега; $U=\{[0, \varepsilon): \varepsilon>0\}$. Легко заметить, что $\varphi-$ неатомарная. Однако $R$ не будет 1-ограниченным в $(R, \Delta, I(\varphi))$.

Замечание 2 . Если $R-$ булева алгебра, то условие исчерпываемости может быть опущено.

В работе [11] доказана следующая теорема.

Теорема 2. Пусть $G$ - топологическая абелева группа, $R$ - кольцо множеств, $\varphi: R \rightarrow G-$ конечно аддитивная исчерпывающая мера. Тогда множество значений меры 2-ограничено.

Эта теорема остается справедливой, если вместо кольца множеств рассмотреть булево кольцо, причем доказательство практически полностью сохраняется. Продолжим исследование внешних мер.

Теорема 3. Если $\varphi: R \rightarrow G-$ исчерпывающая внешняя мера, то множество $R 2$-ограничено в $(R,+, I(\varphi))$.

Доказательство. Рассмотрим тождественное отображение Id: $R \rightarrow(R,+, I(\varphi))$. Ясно, что отображение Id можно рассматривать как конечную аддитивную меру, определенную на булевом кольце $R$ со значениями в топологической абелевой группе $(R,+, I(\varphi))$. Заметим исчерпываемость отображения Id. Действительно, иначе существует окрестность нуля $\tilde{v}$ группы $(R,+, I(\varphi))$ и дизъюнктивная последовательность $\left\{x_{n}\right\} \subset P$ такие, что $\operatorname{Id}\left(x_{n}\right) \notin \tilde{v}$. Это означает, что $x_{n} \notin \tilde{v}$ и существует $z_{n} \leq x_{n}, x_{n} \in R$ со свойством $\varphi\left(z_{n}\right) \notin v$, что противоречит исчерпываемости $\varphi$, так как $\left\{z_{n}\right\}$ также дизъюнктивная. Выполнены все ус- ловия теоремы 2, следовательно, $\operatorname{Id}[R]=R$ будет 2 -ограничено в $(R,+, I(\varphi))$. Теорема доказана.

Из теоремы 1 следует, что внешняя мера $\varphi$ не является неатомарной, если существуют $u \in U$ и $x \in R$ такие, что $x \notin \stackrel{n}{+} \tilde{u}$ для любого $n$. Будем называть такие элементы $\mathcal{u}$-атомами внешней меры $\varphi$. При исследовании понятий ограниченности мы заметили, что для 2-ограниченности множества имеются две возможности: или $Q 1$-ограничено, или $\mathrm{Q} \subset \bigcup_{i=1}^{k}\left(g_{i}+\stackrel{n}{+} u\right)$, где $g_{1}=\theta, g_{i} \notin \stackrel{m}{+} u$ для любого $m$ и $i=2, \ldots, k$, причем $g_{2}, \ldots, g_{k}$ можно выбрать из самого множества $Q$. C помощью введенного понятия атомарности рассмотрим $R$ как подмножество топологической абелевой группы $(R,+, I(\varphi))$ и в результате получаем справедливость следующей теоремы.

Теорема 4. Пусть $\varphi-$ исчерпывающая внешняя мера, тогда для любого $u \in U$ существует лишь конечное множество $u$-атомов.

Заключение. В результате проведенных исследований выявлены важные свойства абстрактных внешних мер. В теоремах 1, 3 и 4 установлено, что понятие неатомарности характеризуется как свойство ограниченности области определения внешней меры в подходящей топологической группе. Данные результаты продолжают исследования Л.Я. Савельева по внешним мерам и топологиям.

\section{Библиографический список}

1. Владимиров Д.А. Булевы алгебры. М., 1969.

2. Савельев Л.Я. Внешние меры и внешние топологии // Сиб. матем. журнал. 1983. № 24 (2),

3. Савельев Л.Я. Продолжение внешних мер // Докл. AH CCCP. 1981. Т. 267. № 4.

4. Бурбаки Н. Общая топология. Основные структуры. M., 1986.

5. Савельев Л.Я. Непрерывные меры // Сиб. электрон. матем. изв. 2008. № 5.

6. Lavrent'ev M.M., Savel'ev L.J. Operator Theory and Ill-Posed Problems. Brill Academic Publishers, Netherlands, Leiden; Martinus Nijhoff Publishers and VSP, Boston, 2006.
7. Срибная Т.А. Критерии равномерной исчерпываемости семейства векторных внешних мер // Вестник СамГУ : Естественнонаучная серия. 2012. № 6 (97).

8. Срибная Т.А. Теорема Брукса-Джеветта о равномерной исчерпываемости на не-сигма-полном классе множеств // Вестник Сам. ун-та : Естественнонаучная серия. 2017. T. 23. № 4.

9. Pap E., Gavrilut A., Agop M. Atomicity via regularity for non-additive set multifunctions // Soft computing. 2016. V. 20.

10. Cavaliere P., Ventriglia F. On nonatomicity for nonadditive functions // J. Math. Anal. Appl. 2014. 415.

11. Кац М.П. О продолжении векторных мер // Сиб. матем. журнал. 1972. Т. 13. № 5. 